\title{
Oxytocin Treatment for Amphetamine-Induced Social Impairments
}

\author{
Dean S. Carson \\ Department of Psychiatry and Behavioral Sciences, Stanford University School of Medicine, Stanford, California 94305 \\ Review of Young et al.
}

The formation and maintenance of enduring social relationships is a critical component of our human condition and has become a major focus of neuroscientific investigation during the past few decades. Research by Cort Pedersen and Arthur Prange in the late 1970s provided initial evidence that oxytocin has a role in social behavior, by showing that central administration of the neuropeptide to virgin female rats results in the expression of maternal behavior (Pedersen and Prange, 1979). There has been a subsequent explosion in the number of preclinical and early clinical studies investigating the involvement of oxytocin in complex social processes. These studies provide convincing evidence that oxytocin attenuates stress and anxiety and improves social cognitive functioning, including emotion recognition, social memory, and trust (MeyerLindenberg et al., 2011). Clinical trials of intranasal oxytocin for the treatment of anxiety, autism, and schizophrenia are currently ongoing and have provided encouraging findings thus far. These studies show that oxytocin reduces cortisol concentrations and subjective and neurobiological responses to psychosocial stress, increases eye-gaze and emotion recognition in autism, and reduces psychotic symptoms and

Received Aug. 21, 2014; revised Sept. 17, 2014; accepted Sept. 30, 2014.

Correspondence should be addressed to Dr. Dean S. Carson, Department of Psychiatry and Behavioral Sciences, Stanford University School of Medicine, 1201 Welch Road, Stanford, CA 94305-5485. E-mail: dcarson@stanford.edu.

DOI:10.1523/JNEUROSCI.3509-14.2014

Copyright $\odot 2014$ the authors $\quad 0270-6474 / 14 / 3414503-03 \$ 15.00 / 0$ improves social functioning in schizophrenia (Bakermans-Kranenburg and van Ijzendoorn, 2013).

In addition to these roles in social behavior, abundant evidence has amassed in support of oxytocin as a therapeutic target in addiction. Zoltán Sarnyai and Gábor Kovács lead innovative research during the 1980-1990s that outlined a role for oxytocin in modulating the physiological and behavioral effects of alcohol, opiates, and psychostimulants (Sarnyai and Kovács, 1994). This preclinical research showed that oxytocin blocks alcohol tolerance and withdrawal, attenuates the development of tolerance to morphine, blocks naloxone-induced morphine withdrawal, reduces heroin self-administration, and decreases cocaineinduced locomotor hyperactivity, stereotyped behavior, and self-administration. This research also provided evidence that oxytocin inhibits dopamine neurotransmission in basal forebrain regions [e.g., posterior olfactory nucleus, tuberculum olfactorium, nucleus accumbens (NAcc), central amygdaloid nucleus, and the hippocampus]. More recent work has shown that oxytocin reduces methamphetamine-induced locomotor hyperactivity, conditioned place preference, and selfadministration, and modulates dopaminergic activity in the NAcc, striatum, medial prefrontal cortex (mPFC), and subthalamic nucleus (for detailed review, see Carson et al., 2013). Furthermore, repeated oxytocin treatment in adolescent alcohol preferring rats reduces alcohol self-administration and anxiety and increases social behavior while producing long-term increases in plasma oxytocin concentrations and upregulating oxytocin receptor (OTR) mRNA in the hypothalamus (Bowen et al., 2011). Importantly, this work is now being translated to early clinical trials in humans that indicate a clear potential for oxytocin as a treatment for addiction (Pedersen et al., 2013).

One of the most exciting prospects for oxytocin as an addiction therapeutic is that it has unparalleled potential for additionally treating associated psychological and social problems. Chronic substance abuse leads to severe psychological issues including anxiety, depression, neurocognitive deficits, and psychosis. Substance abuse is also commonly associated with child abuse and neglect, partner-directed physical and psychological aggression, and high rates of divorce (McGinnis and Foege, 1999). However, the impact of drugs of abuse on social behavior has only recently been investigated at a neurobehavioral level. This research provides strong evidence for drugs of abuse "hijacking" the brain's social reward circuitry. For example, findings implicate altered mesocorticolimbic dopamine activity in drug-induced impairments in partner preference formation in the socially monogamous prairie vole (Microtus ochragaster) (Young et al., 2011a).

Prairie voles are among 3-5\% of mammalian species that form enduring pair bonds after mating and they have become a fascinating model for neurobiological 
investigation and drug development in disorders of social behavior. In fact, research now unequivocally supports the role of oxytocin and the closely related nonapeptide arginine vasopressin (AVP) in the formation of pair bonds in prairie voles. Additionally, pharmacological manipulations in female prairie voles show that blocking dopamine D2-like receptors (D2Rs) in the NAcc, but not the prelimbic cortex (PLC), inhibits the formation of a partner preference after mating, whereas stimulation of D2Rs in the NAcc facilitates the formation of pair bonds in the absence of mating. In contrast, D1-like receptors (D1R) are thought to play a key role in the maintenance of mating-induced partner preference. That is, pair-bonded male prairie voles have significantly higher D1R binding in the NAcc and blocking D1Rs reduces the expression of selective aggression toward female strangers in pair-bonded males (for detailed review, see Young et al., 2011b).

Interestingly, repeated administration of the commonly abused psychostimulant amphetamine in male prairie voles before pairing with a sexually receptive female prevents the formation of a matinginduced partner preference. Treatment of male prairie voles with amphetamine also results in enhanced D1R, but not D2R, expression in the NAcc and pharmacological blockade of NAcc D1Rs rescues mating-induced partner preference in amphetamine-treated animals (Young et al., 2011a). Given the role of D1Rs in the maintenance of mating-induced partner preference, it can therefore be hypothesized that enhanced D1R activity following amphetamine administration is analogous to the animals having formed an enduring bond to the drug reward that then prevents the formation of a pair bond with a mate. This hypothesis is supported by evidence that amphetamine treatment results in the rapid formation of a conditioned place preference for the chamber in which the drug is administered, and is also reminiscent of many drug addicts and alcoholics who fail to develop or maintain intimate relationships.

In work recently published in The Journal of Neuroscience, Young et al. (2014) aimed to determine the shared involvement of the oxytocinergic and dopaminergic systems in amphetamine-mediated impairment of partner preference formation in female prairie voles and to determine the utility of oxytocin as a treatment for drug-induced social deficits. In this research, Young and colleagues (2014) first replicated their previous work in male prairie voles by showing that repeated doses of amphetamine to female prairie voles impaired mating-induced partner preference formation. Next, they provided evidence that repeated amphetamine administration significantly lowered OTR density in the mPFC and significantly lowered D2R density in the NAcc in female voles. No differences in D1R, OTR, or D2R densities were noted in any of the other brain regions analyzed, indicating that the effects of amphetamine on the oxytocinergic and dopaminergic systems are site- and receptor-specific in female prairie voles. In addition, the authors found increased dopamine concentrations in NAcc microdyalisates $24 \mathrm{~h}$ after the final day of repeated amphetamine injections as compared with saline-treated animals. Importantly, all animals, regardless of drug treatment, showed increased NAcc dopamine concentrations during and after mating and amphetamine-treated animals showed a trend toward higher concentrations at all time points compared with saline-treated animals. Given that amphetamine treatment resulted in reduced OTR density in the $\mathrm{MPFC}$ and that previous research indicated the importance of OTR activity in the PLC, an $\mathrm{mPFC}$ subregion, in pair bonding, the researchers showed that infusing oxytocin into the PLC rescued amphetamineinduced deficits in pair bonding. Importantly, coadministration of a selective OTR antagonist blocked the effects of PLC oxytocin infusions, suggesting that this effect is specific to OTRs and not closely related neuropeptide receptor systems [e.g., AVP V1a receptors (AVPR1a)]. Finally, the authors presented evidence that infusing oxytocin into the PLC blocked amphetamine-induced increases in NAcc dopamine to restore mating-induced partner preference formation. Together, these findings provide strong support that amphetamine-induced social deficits in female prairie voles results from selective inhibition of both OTR and $\mathrm{D} 2 \mathrm{R}$ activity in the mesocorticolimbic system and suggest a therapeutic potential for oxytocin in the treatment of drug-induced social deficits.

Recent evidence supports the existence of D2R-OTR heteromers in the ventral and dorsal striatum, thus strongly supporting the assertion that these receptor systems play comodulatory roles in the control of both social and drug related reward (Romero-Fernandez et al., 2013). As previously mentioned, there is also a sub- stantial body of evidence strongly supporting a role of the neuropeptide AVP in social behavior. Importantly, the AVPR1a system is more selectively implicated in the formation of a partner preference in male prairie voles and is also highly influenced by dopaminergic activity (Young et al., 2011b). Thus, it would be interesting to determine how drugs of abuse differentially impact AVPR1a and OTR expression and how this is related to dopaminergic activity in the mesocorticolimbic system to cause deficits in male and female social behavior (Young and Wang, 2004). Regardless of the specific mechanism, translation of these findings to human clinical trials is urgently needed. It is possible that stimulation of the central oxytocinergic system could shift an addict's focus from drug reward to social reward, thus substantially facilitating the recovery process. It is also interesting to consider the therapeutic potential of psychosocial interventions and how they may interact with the endogenous oxytocinergic system. For example, it is possible that the therapeutic effect of 12-step programs such as Alcoholics Anonymous and Narcotics Anonymous, which provide addicts with a group-based social forum for helping each other stay clean and sober, might be driven by the endogenous oxytocinergic system and could also potentially be enhanced by exogenous oxytocin treatment.

\section{References}

Bakermans-Kranenburg MJ, van Ijzendoorn MH (2013) Sniffing around oxytocin: review and meta-analyses of trials in healthy and clinical groups with implications for pharmacotherapy. Transl Psychiatry 3:e258. CrossRef Medline

Bowen MT, Carson DS, Spiro A, Arnold JC, McGregor IS (2011) Adolescent oxytocin exposure causes persistent reductions in anxiety and alcohol consumption and enhances sociability in rats. Plos One 6:e27237. CrossRef Medline

Carson DS, Guastella AJ, Taylor ER, McGregor IS (2013) A brief history of oxytocin and its role in modulating psychostimulant effects. J Psychopharmacol 27:231-247. CrossRef Medline

McGinnis JM, Foege WH (1999) Mortality and morbidity attributable to use of addictive substances in the United States. Proc Assoc Am Physicians 111:109-118. CrossRef Medline

Meyer-Lindenberg A, Domes G, Kirsch P, Heinrichs M (2011) Oxytocin and vasopressin in the human brain: social neuropeptides for translational medicine. Nat Rev Neurosci 12: 524-538. CrossRef Medline

Pedersen CA, Prange AJ Jr (1979) Induction of maternal behavior in virgin rats after intracerebroventricular administration of oxytocin. 
Proc Natl Acad Sci U S A 76:6661-6665. CrossRef Medline

Pedersen CA, Smedley KL, Leserman J, Jarskog LF, Rau SW, Kampov-Polevoi A, Casey RL, Fender T, Garbutt JC (2013) Intranasal oxytocin blocks alcohol withdrawal in human subjects. Alcohol Clin Exp Res 37:484-489. CrossRef Medline

Romero-Fernandez W, Borroto-Escuela DO, Agnati LF, Fuxe K (2013) Evidence for the existence of dopamine d2-oxytocin receptor heteromers in the ventral and dorsal striatum with facilitatory receptor-receptor interactions. Mol Psychiatry 18:849-850. CrossRef Medline

Sarnyai Z, Kovács GL (1994) Role of oxytocin in the neuroadaptation to drugs of abuse. Psychoneuroendocrinology 19:85-117. CrossRef Medline

Young KA, Gobrogge KL, Wang Z (2011a) The role of mesocorticolimbic dopamine in regulating interactions between drugs of abuse and social behavior. Neurosci Biobehav Rev 35: 498-515. CrossRef Medline

Young KA, Gobrogge KL, Liu Y, Wang Z (2011b)
The neurobiology of pair bonding: insights from a socially monogamous rodent. Front Neuroendocrinol 32:53-69. CrossRef Medline

Young KA, Liu Y, Gobrogge KL, Wang H, Wang Z (2014) Oxytocin reverses amphetamineinduced deficits in social bonding: evidence for an interaction with nucleus accumbens dopamine. J Neurosci 34:8499-8506. CrossRef Medline

Young LJ, Wang Z (2004) The neurobiology of pair bonding. Nat Neurosci 7:1048-1054. CrossRef Medline 